\title{
An effective protein extraction method for two-dimensional electrophoresis in the anticancer herb Andrographis paniculata Nees.
}

\begin{abstract}
Proteomic analysis of plants relies on high yields of pure protein. In plants, protein extraction and purification present a great challenge due to accumulation of a large amount of interfering substances, including polysaccharides, polyphenols, and secondary metabolites. Therefore, it is necessary to modify the extraction protocols. A study was conducted to compare four protein extraction and precipitation methods for proteomic analysis. The results showed significant differences in protein content among the four methods. The chloroformtrichloroacetic acid-acetone method using 4-(2-hydroxyethyl)-1-piperazineethanesulfonic acid (HEPES) buffer provided the best results in terms of protein content, pellets, spot resolution, and intensity of unique spots detected. An overall of 83 qualitative or quantitative significant differential spots were found among the four methods. Based on the 2-DE gel map, the method is expected to benefit the development of high-level proteomic and biochemical studies of Andrographis paniculata, which may also be applied to other recalcitrant medicinal plant tissues.
\end{abstract}

Keyword: Andrographis paniculata; Protein extraction; Proteome analysis; Twodimensional electrophoresis. 